\title{
Optimal Packet Scheduling for Delay Minimization in an Energy Harvesting System
}

\author{
Tian Tong ${ }^{1}$, Sennur Ulukus ${ }^{2}$, and Wei Chen ${ }^{1}$ \\ ${ }^{1}$ Department of Electronic Engineering, Tsinghua University \\ ${ }^{2}$ Department of Electrical and Computer Engineering, University of Maryland
}

\begin{abstract}
We consider an energy harvesting communication system, where both energy and data packets arrive at the transmitter during the course of communication. We determine the optimum packet scheduling scheme that minimizes the average delay experienced by all packets. We show that, different from the existing literature, the optimum transmission power is not constant between the energy harvesting and data arrival events; the transmission power starts high, decreases linearly, and potentially reaches zero between energy harvests and data arrivals. Intuitively, untransmitted bits experience cumulative delay due to the bits to be transmitted ahead of them, and hence the reason for transmission power starting high and decreasing over time between energy harvests and data arrivals.
\end{abstract}

\section{INTRODUCTION}

We consider an energy harvesting communication system where the transmitter harvests energy from nature to maintain the power necessary for data transmission. In this system, both energy and data packets arrive at the transmitter during the course of communication, and the transmitter needs to schedule the transmission of the data packets using the available energy. Recent literature considered the optimum packet scheduling problem for this system for the goals of minimizing the transmission completion time [1] and maximizing the throughput [2]-[4]. Reference [1] found the optimum packet scheduling that minimizes the time by which all packets are transmitted. Reference [2] showed the equivalence of this problem to the problem of maximizing throughput by a given time, and extended [1] to the case of a finite-sized battery. Reference [3] generalized [1], [2] to fading channels and developed the directional water-filling algorithm (see also [4]). This line of research has been extended to the case of broadcast channels [5]-[7], multiple access channels [8], interference channels [9], relay channels [10]-[13], and settings with energy cooperation [14]. In addition, energy leakage from battery over time [15], battery inefficiency at the time of charging [16], processing costs [17], [18], and systems with hybrid energy storage [19] have been considered.

In this paper, we consider the problem of average delay minimization in an energy harvesting system. In [1], the problem of minimizing the transmission completion time is considered. Reference [1] and the subsequent literature showed that, due to the concavity of the rate-power relationship, the transmit power must be kept constant between energy harvesting and data arrival events, and the transmitter must schedule data transmissions using longest possible stretches of

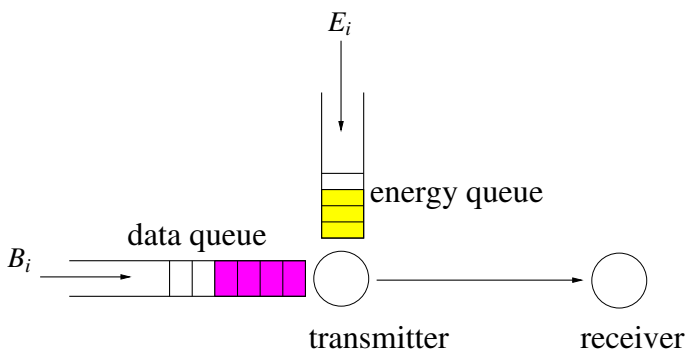

Fig. 1. An energy harvesting communication system.

constant power, subject to energy and data causality. While [1] minimizes the time by which all of the data packets are transmitted, different data packets experience different delays, and the average delay of the system is not minimized. In particular, when the earlier-arriving data packets are transmitted slowly, the later-arriving data packets experience not only the delay in their own transmissions, but a portion of the delay experienced by the earlier-arriving packets, as they have to wait extra time in the data queue while those packets are being transmitted. This compounds the delays that the later-arriving data packets experience. The delay minimization problem was considered previously in [20] for a non energy harvesting system.

We show in this paper that, unlike the previous literature, the transmission power should not be kept constant between energy harvesting and data arrival events. We let the power (and therefore the rate) vary even during the transmission of a single packet. We show that the optimal packet scheduling is such that the transmit power starts with a high value and decreases linearly over time possibly reaching zero before the arrival of the next energy or data packet into the system. The high initial transmit power values ensure that earlier bits are transmitted faster, decreasing their own delay and also the delays of the later-arriving data packets. We develop an iterative algorithm that finds the optimal transmit power over time by determining the optimal Lagrange multipliers.

\section{System Model AND Problem Formulation}

We consider a single-user non-fading energy harvesting communication system, as shown in Fig. 1. The energies are harvested as times $t_{0}^{e}=0, t_{1}^{e}, \ldots, t_{M-1}^{e}$ in amounts $E_{0}, E_{1}, \ldots, E_{M-1}$, respectively, and data packets arrive at times $t_{0}^{b}=0, t_{1}^{b}, \ldots, t_{N-1}^{b}$ in sizes $B_{0}, B_{1}, \ldots, B_{N-1}$, respectively, as shown in Fig. 2. We denote the cumulative harvested 


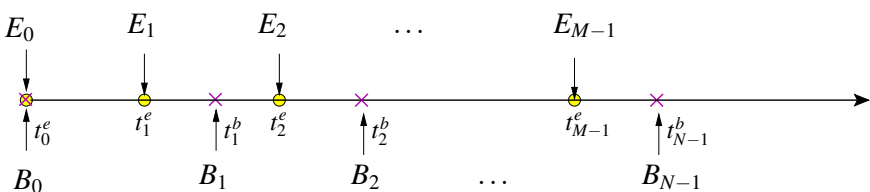

Fig. 2. Energy and data arrivals at the transmitter.

energy and the total number of available bits at time $t$ as:

$$
\begin{aligned}
E_{a}(t) & =\sum_{i=0}^{m-1} E_{i}, \quad t_{m-1}^{e}<t \leq t_{m}^{e}, \quad m=1, \ldots, M \\
B_{a}(t) & =\sum_{j=0}^{n-1} B_{j}, \quad t_{n-1}^{b}<t \leq t_{n}^{b}, \quad n=1, \ldots, N
\end{aligned}
$$

where $t_{M}^{e}$ and $t_{N}^{b}$ are defined as $\infty$. We assume a monotonically increasing concave rate-power relationship $r(p)$, which denotes the rate achieved when power $p$ is used. Many realistic communication schemes result in such rate-power relationships. A typical example is the Gaussian channel capacity formula, $r(p)=\frac{1}{2} \log (1+h p)$, where $h$ represents the (squared) fading. For convenience, we will use $r(p)=\log (1+p)$ for numerical evaluations in this paper.

For a transmission policy, where transmission power at time $t$ is $p(t)$, we define $E(t)$ as the energy consumed up to time $t$, and $B(t)$ as the total number of bits departed up to time $t$ :

$$
\begin{aligned}
& E(t)=\int_{0}^{t} p(\tau) d \tau \\
& B(t)=\int_{0}^{t} r(p(\tau)) d \tau
\end{aligned}
$$

Our objective is to find an optimal power control policy, $p(t)$, in order to minimize the average delay during the transmission of all arriving data packets. The delay for each bit is the time interval from its arrival time to its actual transmission time. The total delay, denoted as $D$, can be calculated as:

$$
D=\int_{0}^{\infty} t d B(t)-\int_{0}^{\infty} t d B_{a}(t)
$$

For a given data arrival profile, the second term in (5) is fixed. Therefore, minimizing $D$ is equivalent to minimizing the gross delay defined as:

$$
D_{g}=\int_{0}^{\infty} t d B(t)=\int_{0}^{\infty} r(p(t)) t d t
$$

We show $D$ and $D_{g}$ as shaded areas in Fig. 3. In the top figure, the green area, which is the area between the data arrival and departure curves, gives the actual delay. The blue area in the bottom figure, is the green area plus the area above the data arrival curve, which is fixed when the data arrival profile is fixed. Therefore, minimizing the blue area (gross delay) is equivalent to minimizing the green area (actual delay). We note that, the gross delay in (6) is the integral of the rate multiplied by time. Intuitively, its minimization will require using small rates, and therefore small powers, for larger times.

Considering the energy and data causality constraints, the
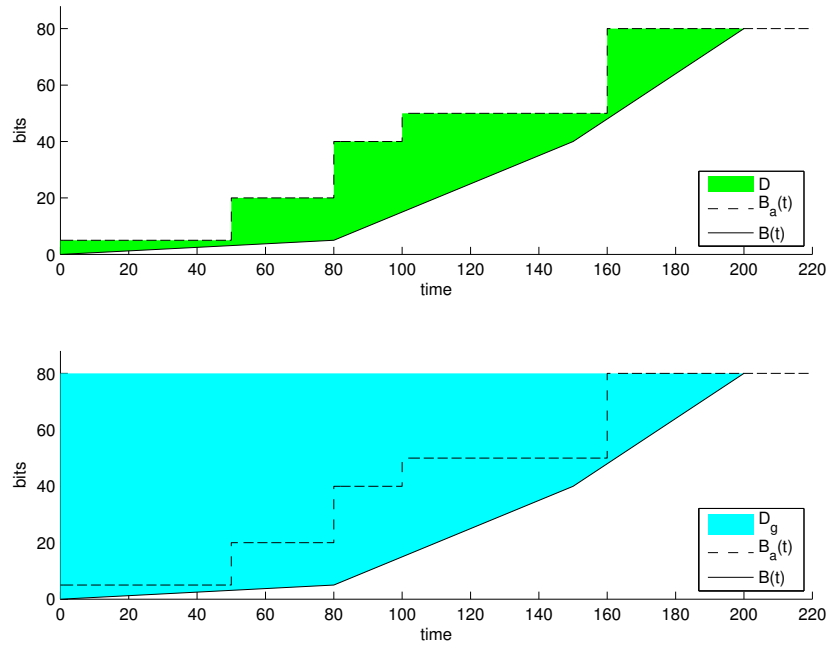

Fig. 3. Illustration of the delay $D$ and the gross delay $D_{g}$.

optimization problem becomes:

$$
\begin{aligned}
\min _{p(t) \geq 0} & D_{g}=\int_{0}^{\infty} r(p(t)) t d t \\
\text { s.t. } & E\left(t_{m}^{e}\right) \leq E_{a}\left(t_{m}^{e}\right), \quad m=1, \ldots, M \\
& B\left(t_{n}^{b}\right) \leq B_{a}\left(t_{n}^{b}\right), \quad n=1, \ldots, N-1 \\
& B\left(t_{N}^{b}\right)=B_{a}\left(t_{N}^{b}\right)
\end{aligned}
$$

where the first two lines in the constraints are due to the energy and data causality constraints, i.e., energy consumed before the $m$ th energy harvesting time cannot exceed the available energy $E_{a}\left(t_{m}^{e}\right)$ and the number of bits transmitted before the $n$th data arrival time cannot exceed the number of available bits $B_{a}\left(t_{n}^{b}\right)$; the third line of the constraint imposes that all incoming data should be transmitted by the end of communication.

\section{Finding the Optimal TRAnSmission Policy}

We divide our development into three parts: First, we determine the optimal power control policy in closed form in terms of two time functions $\lambda(t)$ and $\mu(t)$. Then, we determine the structural properties of $\lambda(t)$ and $\mu(t)$ via recursive relationships. Therefore, the overall solution depends only the initial value of one of these functions. Finally, we determine that optimum initial value through an iterative algorithm.

\section{A. The Optimal Policy in Terms of the Lagrange Multipliers}

In this section, we determine the optimal power control policy in terms of two time-varying parameters through a KKT analysis. The Lagrangian of (7) is given by:

$$
\begin{aligned}
\mathcal{L}= & \int_{0}^{\infty} r(p(t)) t d t+\sum_{m=1}^{M} \lambda_{m}\left(E\left(t_{m}^{e}\right)-E_{a}\left(t_{m}^{e}\right)\right) \\
& +\sum_{n=1}^{N-1} \mu_{n}\left(B\left(t_{n}^{b}\right)-B_{a}\left(t_{n}^{b}\right)\right)-\nu\left(B\left(t_{N}^{b}\right)-B_{a}\left(t_{N}^{b}\right)\right)
\end{aligned}
$$

where $\lambda_{m}, m=1, \ldots, M ; \mu_{n}, n=1, \ldots, N-1$; and $\nu$ are the Lagrange multipliers. 
To find the optimal power $p^{*}(t)$, we take the derivative of $\mathcal{L}$ with respect to $p(t)$. Let $r^{\prime}(p)$ denote the derivative of the function $r(p) \cdot r^{\prime}(p)$ is a positive and monotonically decreasing function since $r(p)$ is increasing and concave. Then, the KKT optimality conditions give:

$$
\left(\nu^{*}-\sum_{\left\{n: t_{n}^{b} \geq t\right\}} \mu_{n}^{*}-t\right) r^{\prime}\left(p^{*}(t)\right)=\sum_{\left\{m: t_{m}^{e} \geq t\right\}} \lambda_{m}^{*}, \forall t
$$

if $p^{*}(t)$ determined by (9) is non-negative, and $p^{*}(t)=0$, otherwise. We use superscript $*$ to denote all optimal values.

We define two time-varying functions $\lambda(t)$ and $\mu(t)$ as:

$$
\begin{aligned}
\lambda(t) & =\sum_{\left\{m: t_{m}^{e} \geq t\right\}} \lambda_{m}^{*} \\
\mu(t) & =\nu^{*}-\sum_{\left\{n: t_{n}^{b} \geq t\right\}} \mu_{n}^{*}
\end{aligned}
$$

We also define a function $f(\cdot)$, which is the inverse function of $1 / r^{\prime}$, with $f: D \rightarrow R^{+} \cup\{0\}$. The function $f(\cdot)$ is positive and monotonically increasing in the domain $D=\left[1 / r^{\prime}(0), \infty\right)$. Finally, we define the function $f^{+}(\cdot)$, with $f^{+}: R \rightarrow R^{+} \cup$ $\{0\}$, as $f^{+}(x)=f(x)$, if $x \in D$, and $f^{+}(x)=0$, if $x \notin D$. The function $f^{+}(\cdot)$ is non-negative and monotonically nondecreasing. With these definitions, $p^{*}(t)$ can be expressed as:

$$
p^{*}(t)=f^{+}\left(\frac{\mu(t)-t}{\lambda(t)}\right)
$$

For the special case of $r(p)=\log (1+p)$, it can be verified that we have $f^{+}(x)=(x-1)^{+}$.

\section{B. Properties and Recursive Construction of $\lambda(t)$ and $\mu(t)$}

In the previous section, we showed that the optimal power policy is determined only by two functions $\mu(t)$ and $\lambda(t)$. In this section, we provide properties of $\mu(t)$ and $\lambda(t)$, and also a procedure to construct them recursively, once an initial value of one of them is given. We will give an algorithm to find the optimal such initial value in the next section.

We first state in Lemma 1 that $\lambda(t)$ and $\mu(t)$ are piecewise constant, and only potentially change at time instants where an energy or a data causality constraint is met with equality. Therefore, the time instants where $\lambda(t)$ and $\mu(t)$ change can be divided into two types: type- $E$ changes for harvesting time instants $t_{m}^{e}$ where $E^{*}\left(t_{m}^{e}\right)=E_{a}\left(t_{m}^{e}\right)$; and type- $B$ changes for data arrival time instants $t_{n}^{b}$ where $B^{*}\left(t_{n}^{b}\right)=B_{a}\left(t_{n}^{b}\right)$.

Lemma 1 In the optimal policy, the changes of $\lambda(t)$ or $\mu(t)$ only occur at some type-E or type-B time instants. The pattern of change is: $\lambda(t)$ decreases after a type-E time instant; $\mu(t)$ increases after a type-B time instant.

Proof: The optimal Lagrange multipliers $\lambda_{m}^{*}$ and $\mu_{n}^{*}$ satisfy the complementary slackness conditions. For $m=1, \ldots, M$,

$$
\begin{aligned}
& \lambda_{m}^{*}=0, \quad \text { if } E^{*}\left(t_{m}^{e}\right)<E_{a}\left(t_{m}^{e}\right) \\
& E^{*}\left(t_{m}^{e}\right)=E_{a}\left(t_{m}^{e}\right), \quad \text { if } \lambda_{m}^{*}>0
\end{aligned}
$$

and similarly, for $n=1, \ldots, N-1$,

$$
\begin{aligned}
& \mu_{n}^{*}=0, \quad \text { if } B^{*}\left(t_{n}^{b}\right)<B_{a}\left(t_{n}^{b}\right) \\
& B^{*}\left(t_{n}^{b}\right)=B_{a}\left(t_{n}^{b}\right), \quad \text { if } \mu_{n}^{*}>0
\end{aligned}
$$

Using the definition of $\lambda(t)$ in (10), and since $\lambda_{m}^{*} \geq 0, \lambda(t)$ decreases only at $t_{m}^{e}$ where $\lambda_{m}^{*}>0$. From (13), $E^{*}\left(t_{m}^{e}\right)=$ $E_{a}\left(t_{m}^{e}\right)$ for such $t_{m}^{e}$, i.e., this is a type-E time instant. A similar argument applies to $\mu(t)$.

From Lemma 1, $\lambda(t)$ and $\mu(t)$ can be expressed by a series of numbers, instead of continuous functions of time. We mark the time instants where either $\lambda(t)$ or $\mu(t)$ changes as an increasing sequence: $t_{1}^{c}, t_{2}^{c}, \ldots, t_{L}^{c}$, and let $\lambda_{k}^{c}$ and $\mu_{k}^{c}$ denote the values of $\lambda(t)$ and $\mu(t)$ after $t_{k}^{c}$ ( $t_{0}^{c}$ defined as 0 ), i.e.,

$$
\lambda(t)= \begin{cases}\lambda_{k}^{c}, & t \in\left(t_{k}^{c}, t_{k+1}^{c}\right], k=0, \ldots, L-1 \\ \lambda_{L}^{c}, & t \in\left(t_{L}^{c}, \infty\right)\end{cases}
$$

and

$$
\mu(t)= \begin{cases}\mu_{k}^{c}, & t \in\left(t_{k}^{c}, t_{k+1}^{c}\right], k=0, \ldots, L-1 \\ \mu_{L}^{c}, & t \in\left(t_{L}^{c}, \infty\right)\end{cases}
$$

With these definitions, Lemma 1 states that:

$$
\begin{aligned}
& \lambda_{k}^{c}=\lambda_{k-1}^{c}, \quad \text { if } E^{*}\left(t_{k}^{c}\right)<E_{a}\left(t_{k}^{c}\right) \\
& E^{*}\left(t_{k}^{c}\right)=E_{a}\left(t_{k}^{c}\right), \quad \text { if } \lambda_{k}^{c}<\lambda_{k-1}^{c}
\end{aligned}
$$

and

$$
\begin{aligned}
& \mu_{k}^{c}=\mu_{k-1}^{c}, \quad \text { if } B^{*}\left(t_{k}^{c}\right)<B_{a}\left(t_{k}^{c}\right) \\
& B^{*}\left(t_{k}^{c}\right)=B_{a}\left(t_{k}^{c}\right), \quad \text { if } \mu_{k}^{c}>\mu_{k-1}^{c}
\end{aligned}
$$

for $k=1, \ldots, L-1$. That is, $\lambda_{k}^{c}$ remains unchanged if the energy queue is not empty, and if $\lambda_{k}^{c}$ changes then it decreases and the energy queue is empty. Similarly, $\mu_{k}^{c}$ remains unchanged if the data queue is not empty, and if $\mu_{k}^{c}$ changes then it increases and the data queue is empty.

We now begin to develop a recursive relationships for $\lambda_{k}^{c}$, $\mu_{k}^{c}$, and $t_{k}^{c}$. Lemma 2 provides a method to derive $t_{k+1}^{c}$ from $t_{k}^{c}$ and $\mu_{k}^{c}$ from $\lambda_{k}^{c}$. Similarly, Lemma 3 provides a method to derive $t_{k+1}^{c}$ from $t_{k}^{c}$ and $\lambda_{k}^{c}$ from $\mu_{k}^{c}$. Therefore, starting from $t_{0}^{c}=0$ and having either $\lambda_{0}^{c}$ or $\mu_{0}^{c}$, and using the recursive relationships in Lemma 2 and Lemma 3, together with the relationships in (17)-(18), we can construct the optimal $\lambda(t)$ and $\mu(t)$ functions, and find the optimal power policy from (12). We will discuss finding the optimal $\mu_{0}^{c}$ in the next section.

Lemma 2 When $E^{*}\left(t_{k}^{c}\right), B^{*}\left(t_{k}^{c}\right)$ and $\lambda_{k}^{c}$ are known at a (typeB) $t_{k}^{c}<\infty$, we have:

$$
\begin{aligned}
\mu_{k}^{c}= & \min \Pi \\
t_{k+1}^{c}= & \arg \min _{\left\{t_{m}^{e}, t_{n}^{b}>t_{k}^{c}\right\}} \Pi \\
\Pi= & \left\{\mu_{m}^{e} ; \mu_{n}^{b} \quad m, n \text { s.t. } t_{m}^{e}, t_{n}^{b}>t_{k}^{c}:\right. \\
& E^{*}\left(t_{k}^{c}\right)+\int_{t_{k}^{c}}^{t_{m}^{e}} f^{+}\left(\frac{\mu_{m}^{e}-t}{\lambda_{k}^{c}}\right) d t=E_{a}\left(t_{m}^{e}\right) \\
& \left.B^{*}\left(t_{k}^{c}\right)+\int_{t_{k}^{c}}^{t_{n}^{b}} r\left(f^{+}\left(\frac{\mu_{n}^{b}-t}{\lambda_{k}^{c}}\right)\right) d t=B_{a}\left(t_{n}^{b}\right)\right\}
\end{aligned}
$$


Proof: First, we prove that such $\mu_{m}^{e}$ and $\mu_{n}^{b}$ in $\Pi$ exist. This is equivalent to proving that (19)-(20) have solutions. Note that $\int_{t_{k}^{c}}^{t_{m}^{e}} f^{+}\left(\frac{\mu_{m}^{e}-t}{\lambda_{k}^{c}}\right) d t$ and $\int_{t_{k}^{c}}^{t_{n}^{b}} r\left(f^{+}\left(\frac{\mu_{n}^{b}-t}{\lambda_{k}^{c}}\right)\right) d t$ are continuous functions of $\mu_{m}^{e}$ or $\mu_{n}^{b} \in\left(t_{k}^{c}, \infty\right)$, which are monotonically increasing and ranging in $(0, \infty)$. Their continuity guarantees the existence of corresponding solutions.

Next, we prove that $\mu_{k}^{c}=\min \Pi$ by contradiction. If $\mu_{k}^{c}>\min \Pi$, then one of the causality constraints at time arg $\min _{\left\{t_{m}^{e}, t_{n}^{b}>t_{k}^{c}\right\}} \Pi$ is violated. If $\mu_{k}^{c}<\min \Pi$, then $\mu(t), \lambda(t)$ will not change after $t_{k}^{c}$ and none of the constraints after $t_{k}^{c}$ will be met with equality, which contradicts with $B^{*}\left(t_{N}^{b}\right)=B_{a}\left(t_{N}^{b}\right)$. Finally, we declare that $t_{k+1}^{c}=$ $\arg \min _{\left\{t_{m}^{e}, t_{n}^{b}>t_{k}^{c}\right\}} \Pi$, for it is the first time after $t_{k}^{c}$ where the causality constraints are met.

Lemma 3 When $E^{*}\left(t_{k}^{c}\right), B^{*}\left(t_{k}^{c}\right)$ and $\mu_{k}^{c}$ are known at a (typeE) $t_{k}^{c}<\mu_{k}^{c}$, we have:

$$
\begin{aligned}
\lambda_{k}^{c}= & \max \Lambda \\
t_{k+1}^{c}= & \arg \max _{\left\{t_{m}^{e}, t_{n}^{b}>t_{k}^{c}\right\}} \Lambda \\
\Lambda= & \left\{\lambda_{m}^{e} ; \lambda_{n}^{b} \quad m, n \text { s.t. } t_{m}^{e}, t_{n}^{b}>t_{k}^{c}:\right. \\
& E^{*}\left(t_{k}^{c}\right)+\int_{t_{k}^{c}}^{t_{m}^{e}} f^{+}\left(\frac{\mu_{k}^{c}-t}{\lambda_{m}^{e}}\right) d t=E_{a}\left(t_{m}^{e}\right) \\
& \left.B^{*}\left(t_{k}^{c}\right)+\int_{t_{k}^{c}}^{t_{n}^{b}} r\left(f^{+}\left(\frac{\mu_{k}^{c}-t}{\lambda_{n}^{b}}\right)\right) d t=B_{a}\left(t_{n}^{b}\right)\right\}
\end{aligned}
$$

The proof of Lemma 3 is similar to that of Lemma 2, which we omit here due to space limitations. Relying on these two lemmas, we can construct the optimal solution if only an initial value of $\lambda(t)$ or $\mu(t)$ is given. We put forth Algorithm 1 to construct the optimal policy with a given value of $\mu_{0}^{c}$.

\section{Determining the Optimal Value of $\mu_{0}^{c}$}

In this section, we propose a method to determine the optimal initial value $\mu_{0}^{c}$. We first note that, in the optimal solution, we must have $B^{*}(\infty)=B_{a}(\infty)$, i.e., all the data packets must be transmitted. This comes from the third line of the constraint set in (7); policies that violate this are not even feasible let alone be optimal. Analogous to this, in Lemma 4, we show that in the optimal solution, we must have $E^{*}(\infty)=E_{a}\left(\mu_{L}^{c}\right)$, i.e., if the last binding constraint is the data constraint, then the final energy must be determined by that. We remark here that $E^{*}(\infty)<E_{a}(\infty)$ is possible, i.e., all of the incoming energy is not always used (this is unlike data, where all of the incoming data is always transmitted and we must always have $B^{*}(\infty)=B_{a}(\infty)$ ). An intuitive explanation for this is that an energy coming too late should not be used in a delay-minimal transmission policy.

Lemma $4 E^{*}(\infty)=E_{a}\left(\mu_{L}^{c}\right)$, where $\mu_{L}^{c}$ is the value of $\mu(t)$ for $t \in\left(t_{L}^{c}, \infty\right)$, i.e., the value for the last period.

Proof: We first note that $E^{*}(t)$ will stay constant after $\mu_{L}^{c}$, i.e., $p^{*}(t)=f^{+}\left(\frac{\mu(t)-t}{\lambda(t)}\right)=0$, for all $t>\mu_{L}^{c} \geq \mu(t)$.

\section{Algorithm 1 Construct a policy with an initial value}

Input: $\left\{t_{m}^{e}\right\}$ : energy harvesting time instants; $\left\{E_{m}\right\}$ : energy

harvesting amounts; $\left\{t_{n}^{b}\right\}$ : bits arrival time instants; $\left\{B_{n}\right\}$ : bits arrival amounts; $\mu_{0}^{c}$ : initial value of $\mu(t)$;

Output: $\left\{t_{k}^{c}\right\}$ : time instants of changes; $\left\{\vartheta_{k}\right\}$ : types of $\left\{t_{k}^{c}\right\}$; $\left\{\mu_{k}^{c}\right\}$ : values of $\mu(t) ;\left\{\lambda_{k}^{c}\right\}$ : values of $\lambda(t)$;

Set $t_{0}^{c} \leftarrow 0 ; k \leftarrow 0 ; \vartheta_{0} \leftarrow$ type-E; $E_{t} \leftarrow 0 ; B_{t} \leftarrow 0$;

while $\vartheta_{k}=$ type-B and $t_{k}^{c}<\infty$, or $t_{k}^{c}<\mu_{k}^{c}$ do

if $\vartheta_{k}=$ type-E then

for all $t_{m}^{e}, t_{n}^{b}>t_{k}^{c}$ do

Solve $\lambda_{m}^{e}, \lambda_{n}^{b}$ from

$$
\begin{aligned}
& E_{t}+\int_{t_{m}^{c}}^{t_{m}^{e}} f^{+}\left(\frac{\mu_{k}^{c}-t}{\lambda_{m}^{e}}\right) d t=\sum_{i=0}^{m-1} E_{i}, \\
& B_{t}+\int_{t_{k}^{c}}^{t_{n}^{b}} r\left(f^{+}\left(\frac{\mu_{k}^{c}-t}{\lambda_{n}^{b}}\right)\right) d t=\sum_{j=0}^{n-1} B_{j} ;
\end{aligned}
$$

\section{end for}

Set $\lambda_{k}^{c} \leftarrow \max \left\{\lambda_{m}^{e}, \lambda_{n}^{b}\right\}$;

Set $t_{k+1}^{c} \leftarrow \arg \max _{\left\{t_{m}^{e}, t_{n}^{b}\right\}}\left\{\lambda_{m}^{e}, \lambda_{n}^{b}\right\}$;

else $\left[\vartheta_{k}=\right.$ type-B]

for all $t_{m}^{e}, t_{n}^{b}>t_{k}^{c}$ do

Solve $\mu_{m}^{e}, \mu_{n}^{b}$ from

$$
\begin{aligned}
& E_{t}+\int_{t_{k}^{c}}^{t_{m}^{e}} f^{+}\left(\frac{\mu_{m}^{e}-t}{\lambda_{k}^{c}}\right) d t=\sum_{i=0}^{m-1} E_{i}, \\
& B_{t}+\int_{t_{k}^{c}}^{t_{n}^{b}} r\left(f^{+}\left(\frac{\mu_{n}^{b}-t}{\lambda_{k}^{c}}\right)\right) d t=\sum_{j=0}^{n-1} B_{j} ;
\end{aligned}
$$

\section{end for}

Set $\mu_{k}^{c} \leftarrow \min \left\{\mu_{m}^{e}, \mu_{n}^{b}\right\}$;

end if

Set $t_{k+1}^{c} \leftarrow \arg \min _{\left\{t_{m}^{e}, t_{n}^{b}\right\}}\left\{\mu_{m}^{e}, \mu_{n}^{b}\right\}$;

if energy constraint is met with equality at $t_{k+1}^{c}$ then

Set $\vartheta_{k+1} \leftarrow$ type-E;

Set $\mu_{k+1} \leftarrow \mu_{k}$;

else [data constraint is met with equality at $t_{k+1}^{c}$ ]

Set $\vartheta_{k+1} \leftarrow$ type-B;

Set $\lambda_{k+1} \leftarrow \lambda_{k}$;

end if

Set $E_{t} \leftarrow E_{t}+\int_{t_{k}^{c}}^{t_{k+1}^{c}} f^{+}\left(\frac{\mu_{k}^{c}-t}{\lambda_{k}^{c}}\right) d t$;

Set $B_{t} \leftarrow B_{t}+\int_{t_{k}^{c}}^{t_{k+1}^{c}} r\left(f^{+}\left(\frac{\mu_{k}^{c}-t}{\lambda_{k}^{c}}\right)\right) d t$;

Set $k \leftarrow k+1$;

\section{end while}

Next, we show that $\mu_{L}^{c}$ cannot be later than the last time instant where the energy constraint is met with equality, i.e.,

$$
\mu_{L}^{c} \leq t_{l}^{e}, l=\max \left\{m \in[1, M]: E^{*}\left(t_{m}^{e}\right)=E_{a}\left(t_{m}^{e}\right)\right\}
$$

If $t_{l}^{e}=\infty$, then (23) is correct. Otherwise, since $\lambda_{m}^{*}=0$ for all $m>l$, we have $\lambda(t)=0$ for all $t>t_{l}^{e}$, given by (10). If $\mu_{L}^{c}>t_{l}^{e}$, then $p^{*}(t)=\infty$ for $t \in\left(t_{l}^{e}, \mu_{L}^{c}\right)$, which is impossible in the optimal policy. Thus, (23) is correct.

Next, we note that $\mu_{L}^{c}>t_{l-1}^{e}$. If $\mu_{L}^{c} \leq t_{l-1}^{e}$, then $E^{*}(t)$ is constant after $t_{l-1}^{e}$, and $E^{*}\left(t_{l}^{e}\right)=E^{*}\left(t_{l-1}^{e}\right) \leq E_{a}\left(t_{l-1}^{e}\right)<$ $E_{a}\left(t_{l}^{e}\right)$, which contradicts with the definition of $l$.

In conclusion, we can deduce that $E_{a}\left(\mu_{L}^{c}\right)=E_{a}\left(t_{l}^{e}\right)$ since $\mu_{L}^{c} \in\left(t_{l-1}^{e}, t_{l}^{e}\right]$, and $E^{*}(\infty)=E^{*}\left(t_{l}^{e}\right)=E^{*}\left(\mu_{L}^{c}\right)$ since $E^{*}(t)$ is constant after $\mu_{L}^{c}$. Therefore, we must have 
$E^{*}(\infty)=E^{*}\left(t_{l}^{e}\right)=E_{a}\left(t_{l}^{e}\right)=E_{a}\left(\mu_{L}^{c}\right)$.

Therefore, from Lemma 4, we know that if we started with a $\mu_{0}^{c}$, which yielded an $E^{*}(\infty)$, which does not satisfy $E^{*}(\infty)=E_{a}\left(\mu_{L}^{c}\right)$, then this $\mu_{0}^{c}$ cannot be optimal. Similarly, if we started with a $\mu_{0}^{c}$, which yielded a $B^{*}(\infty)$, which does not satisfy $B^{*}(\infty)=B_{a}(\infty)$, then this $\mu_{0}^{c}$ cannot be optimal, either. Lemma 5 identifies that if the chosen $\mu_{0}^{c}$ is smaller than the actual optimal value then the required final data constraint will be violated, and if the chosen $\mu_{0}^{c}$ is larger than the actual optimal value then the required final energy constraint will be violated. We will then propose a bisection algorithm to find the optimal $\mu_{0}^{c}$ iteratively. The proof of Lemma 5 is omitted here due to space limitations.

Lemma 5 If Algorithm 1 is executed with a $\mu_{0}^{c}$ which is smaller than the actual optimal value, then we will have:

$$
E(\infty)=E_{a}\left(\mu_{L}^{c}\right), \quad B(\infty)<B_{a}(\infty)
$$

and if it is executed with a $\mu_{0}^{c}$ which is larger than the actual optimal value, then we will have:

$$
E(\infty)<E_{a}\left(\mu_{L}^{c}\right), \quad B(\infty)=B_{a}(\infty)
$$

We next note that we have the lower bound of $\mu_{0}^{c}=0$. In addition, we can have a large enough upper bound for $\mu_{0}^{c}$. We run Algorithm 1 starting with a $\mu_{0}^{c}$ which lies between these bounds. We then use Lemma 5 to determine whether the $\mu_{0}^{c}$ used is smaller or larger than the actual optimal value. We use a bisection method to adjust $\mu_{0}^{c}$ iteratively until we reach the optimal solution. In fact, this procedure is equivalent to solving a single-variable equation for $\mu_{0}^{c}$ by a bisection method.

\section{NuMERICAL RESUlTS}

In this section, we give simple numerical results to validate and further explain the presented results. Throughout this section, we use the rate-power relationship $r(p)=\log (1+p)$.

First, we give an example run of Algorithm 1 in Fig. 4. For this system, energies are harvested at times $[0,20]$ in amounts $[5,15]$, and data packets arrive at times $[0,35]$ in sizes $[10,5]$. Note that throughout the numerical results section, energy harvesting and data arrival numbers are chosen for example purposes and for convenience in plotting, and the actual units should be adjusted accordingly. For this run, we choose $\mu_{0}^{c}=35$, which may not be optimum. From Lemma 3, we first solve $\Lambda=\left\{\lambda_{1}^{e}, \lambda_{2}^{e}, \lambda_{1}^{b}, \lambda_{2}^{b}\right\}$ related to time instants $t_{1}^{e}, t_{2}^{e}, t_{1}^{b}, t_{2}^{b}$ and then derive $\lambda_{0}^{c}=\min \Lambda=\lambda_{1}^{e}=20.6535$, $t_{1}^{c}=t_{1}^{e}=20$. Since $B\left(t_{1}^{c}\right)<B_{a}\left(t_{1}^{c}\right)$, we have $\mu_{1}^{c}=\mu_{0}^{c}=35$ from (18). Next, we similarly derive $\lambda_{1}^{c}=5.3179$ and $t_{2}^{c}=35$. From (17), we have $\lambda_{2}^{c}=\lambda_{1}^{c}$, since $E\left(t_{2}^{c}\right)<E_{a}\left(t_{2}^{c}\right)$. Finally, we obtain $\mu_{2}^{c}=48.4292$ at $t_{3}^{c}=\infty$ according to Lemma 2 . The algorithm comes to an end here. We note that the solution is incorrect, since not all bits are transmitted. That is because the initial value $\mu_{0}^{c}$ was incorrect.

Next, we demonstrate Lemma 5 with a specific example in Fig. 5. Here, we plot three runs of Algorithm 1 with three different initial $\mu_{0}^{c}$ values. For this example, energies are harvested at times $[0,20]$ in amounts $[5,15]$, and data packets
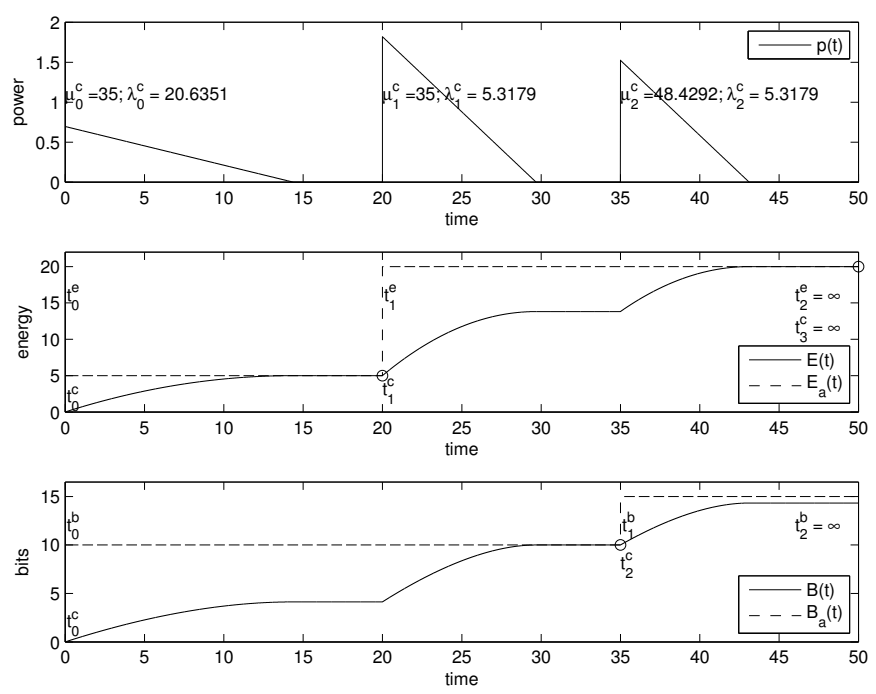

Fig. 4. Example run of Algorithm 1 for a fixed $\mu_{0}^{c}$.

arrive at times $[0,40]$ in sizes $[10,5]$. For this system, the actual optimal $\mu_{0}^{c}$ is approximately $\mu_{0}^{c}=40$. The run on the left uses $\mu_{0}^{c}=35$, which is smaller than the actual optimal value, and therefore, yields $B(\infty) \neq B_{a}(\infty)$, i.e., not all data packets are transmitted. The run on the right uses $\mu_{0}^{c}=50$, which is larger than the actual optimal value, and therefore, yields $E(\infty) \neq E_{a}\left(\mu_{L}^{c}\right)$.

Finally, we consider the example system where the energies are harvested at times $[0,30,80,110,180,230]$ in amounts $[5,10,50,10,50,10]$, and data packets arrive at times $[0,50,90,100,160]$ in sizes $[8,10,22,10,30]$, and run Algorithm 1 together with the bisection algorithm to obtain the optimum power allocation policy. We plot the optimum power allocation policy and the associated energy consumption and data departure curves in Fig. 6. For this setting, we also solve the transmission completion time minimization problem in [1] and plot the corresponding optimum power allocation policy and the associated energy consumption and data departure curves in Fig. 7. We note that our delay minimization power control policy gives a gross delay of $D_{g}=9938$ and a transmission completion time of $T=203$, whereas the transmission completion time minimization policy of [1] gives a gross delay of $D_{g}=10344$ and a transmission completion time of $T=191$. This verifies the difference of the two objectives, difference of the obtained optimum power control strategies, and how each algorithm outperforms the other with respect to its own optimality criteria.

\section{Conclusions}

We considered delay minimization in an energy harvesting communication channel where energy and data packets become available at the transmitter during the course of communication. We determined the optimum power control policy in terms of the Lagrange multiplier functions. We identified the properties of these functions and gave a method that evaluates them recursively. We proposed an iterative algorithm which iteratively updates the initial value of a Lagrange multiplier, 

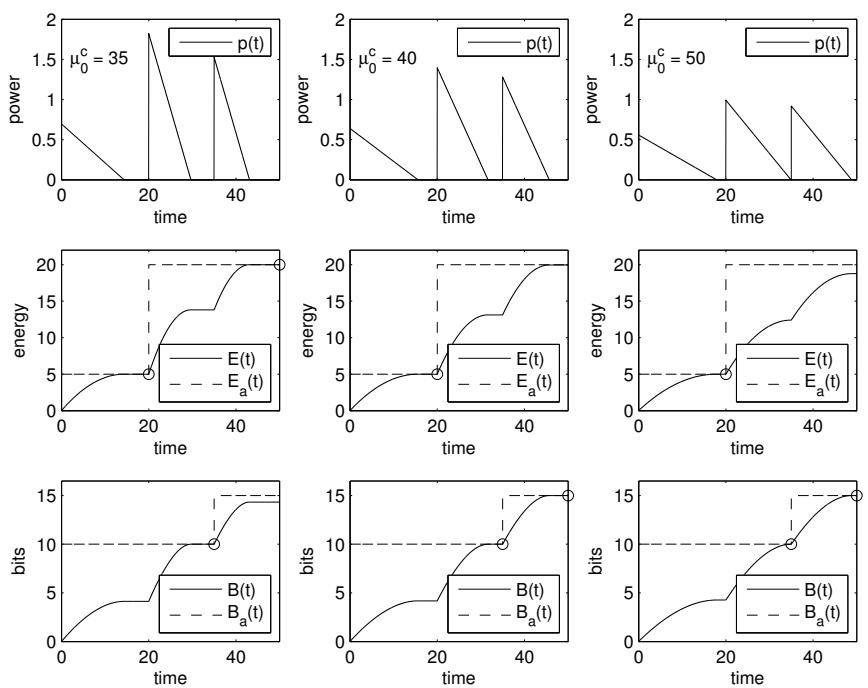

Fig. 5. Comparison of solutions by Algorithm 1 with three different $\mu_{0}^{c}$.

and obtains the optimum power allocation policy. The optimal power values start high, decrease linearly, potentially reaching zero between energy harvests and data arrivals. This policy is different from the piecewise constant power policies of the existing literature which focus on minimizing a deadline by which all packets are transmitted or maximizing the throughput before a fixed deadline. Initial high powers in our case make sure that the delay does not accumulate by transmitting data at faster rates first, then decreasing the rate gradually.

\section{REFERENCES}

[1] J. Yang and S. Ulukus. Optimal packet scheduling in an energy harvesting communication system. IEEE Trans. Comm., 60(1):220-230, January 2012.

[2] K. Tutuncuoglu and A. Yener. Optimum transmission policies for battery limited energy harvesting nodes. IEEE Trans. Wireless Comm. 11(3):1180-1189, March 2012.

[3] O. Ozel, K. Tutuncuoglu, J. Yang, S. Ulukus, and A. Yener. Transmission with energy harvesting nodes in fading wireless channels: Optimal policies. IEEE JSAC, 29(8):1732-1743, September 2011.

[4] C. K. Ho and R. Zhang. Optimal energy allocation for wireless communications with energy harvesting constraints. IEEE Trans. Signal Proc., 60(9):4808-4818, September 2012.

[5] J. Yang, O. Ozel, and S. Ulukus. Broadcasting with an energy harvesting rechargeable transmitter. IEEE Trans. Wireless Comm., 11(2):571-583, February 2012.

[6] M. A. Antepli, E. Uysal-Biyikoglu, and H. Erkal. Optimal packet scheduling on an energy harvesting broadcast link. IEEE JSAC, 29(8):1721-1731, September 2011.

[7] O. Ozel, J. Yang, and S. Ulukus. Optimal broadcast scheduling for an energy harvesting rechargeable transmitter with a finite capacity battery. IEEE Trans. Wireless Comm., 11(6):2193-2203, June 2012.

[8] J. Yang and S. Ulukus. Optimal packet scheduling in a multiple access channel with energy harvesting transmitters. Journal of Comm. and Netw., 14:140-150, April 2012.

[9] K. Tutuncuoglu and A. Yener. Sum-rate optimal power policies for energy harvesting transmitters in an interference channel. Journal of Comm. and Netw., 14(2):151-161, April 2012.

[10] C. Huang, R. Zhang, and S. Cui. Throughput maximization for the Gaussian relay channel with energy harvesting constraints. IEEE JSAC, 31:1469-1479, August 2013

[11] D. Gunduz and B. Devillers. Two-hop communication with energy harvesting. In IEEE CAMSAP, December 2011.

[12] O. Orhan and E. Erkip. Optimal transmission policies for energy harvesting two-hop networks. In CISS, March 2012
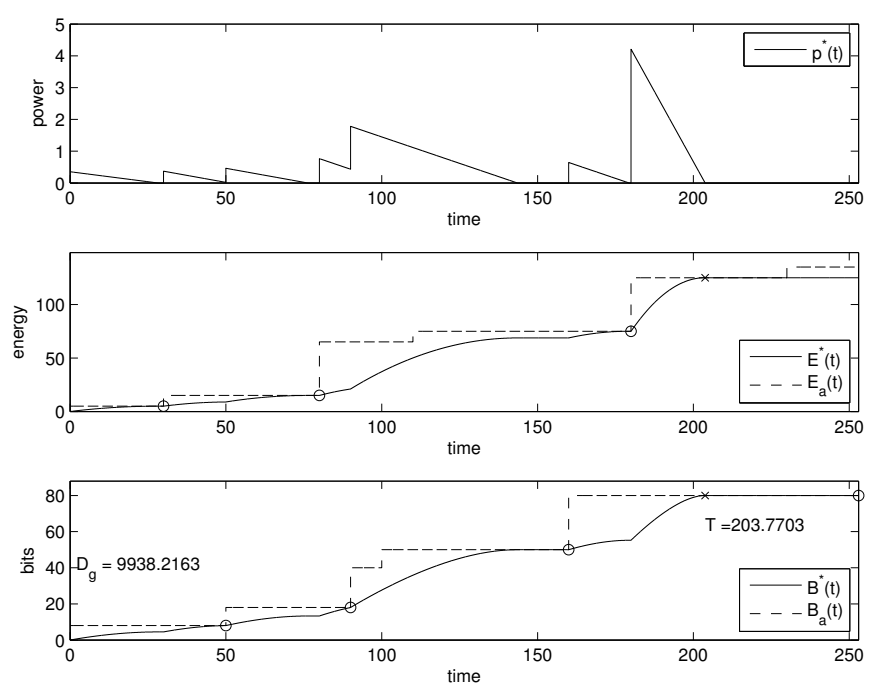

Fig. 6. Visualization of the delay minimal policy.
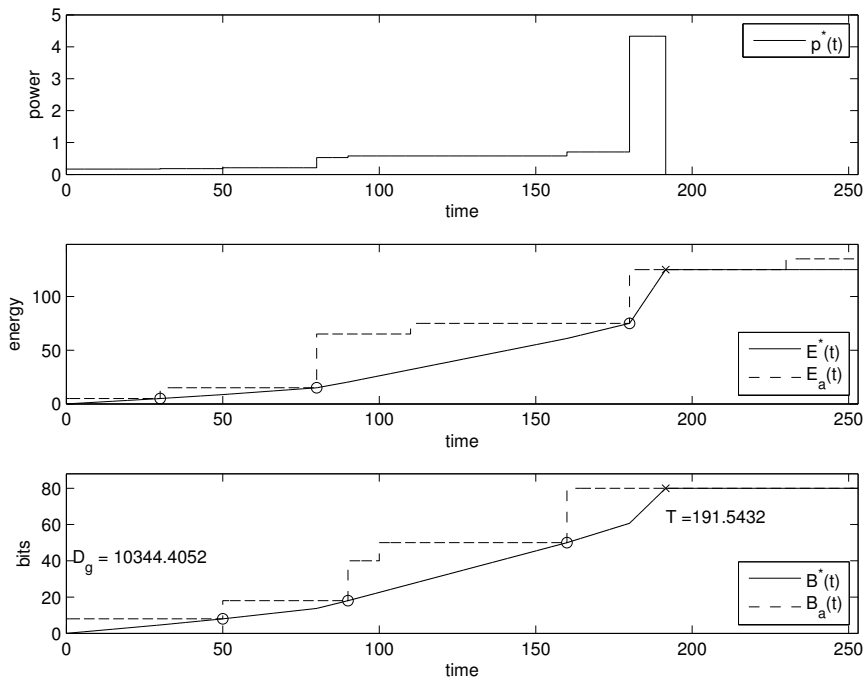

Fig. 7. Visualization of the transmission time minimal policy.

[13] Y. Luo, J. Zhang, and K. B. Letaief. Optimal scheduling and power allocation for two-hop energy harvesting communication systems. IEEE Trans. Wireless Comm., 12(9):4729-4741, September 2013.

[14] B. Gurakan, O. Ozel, J. Yang, and S. Ulukus. Energy cooperation in energy harvesting communications. IEEE Trans. Comm., 61(12):48844898, December 2013.

[15] B. Devillers and D. Gunduz. A general framework for the optimization of energy harvesting communication systems with battery imperfections. Journal of Comm. and Netw., 14(2):130-139, April 2012.

[16] K. Tutuncuoglu and A. Yener. Optimal power policy for energy harvesting transmitters with inefficient energy storage. In CISS, March 2012.

[17] O. Orhan, D. Gunduz, and E. Erkip. Throughput maximization for an energy harvesting communication system with processing cost. In IEEE ITW, September 2012.

[18] J. Xu and R. Zhang. Throughput optimal policies for energy harvesting wireless transmitters with non-ideal circuit power. IEEE JSAC, 32(2):322-332, February 2014.

[19] O. Ozel, K. Shahzad, and S. Ulukus. Optimal energy allocation for energy harvesting transmitters with hybrid energy storage and processing cost. IEEE Trans. Signal Proc., 62(12):3232-3245, June 2014.

[20] J. Yang and S. Ulukus. Delay-minimal transmission for energy constrained wireless communications. In IEEE ICC, May 2008. 